\title{
Cancer research in Brazil - stuck in second gear?
}

\author{
A.P. Lepique ${ }^{1}$ and C. Bonorino ${ }^{2}$ \\ ${ }^{1}$ Departamento de Imunologia, Instituto de Ciências Biomédicas, Universidade de São Paulo, São Paulo, SP, Brasil \\ ${ }^{2}$ Instituto de Pesquisas Biomédicas, Pontifícia Universidade Católica do Rio Grande do Sul, Porto Alegre, RS, Brasil
}

\begin{abstract}
This article describes the main issues regarding clinical cancer research in Brazil, including both the opportunities and the hurdles. Scientists and clinicians in this field had the opportunity to talk to regulatory agencies and to the Health Ministry representative at a meeting held in the State of Rio de Janeiro, Brazil, in April 2014. Our conclusions are that we do indeed have opportunities; however, we need to move forward regarding partnerships between academia and industry, increase the availability of funding, and provide easier navigation through the regulatory processes.
\end{abstract}

Key words: Cancer research; Clinical research; Regulatory agencies; Health Ministry

In April 2014, the beautiful town of Mangaratiba hosted the meeting Challenges and Solutions in Cancer: Research and Treatment. The meeting gathered representatives of major groups working on cancer research in Brazil, both basic and clinical. Researchers were from the National Institute for Cancer (INCA), the Institute of Cancer from the State of São Paulo, Sirio-Libanês Hospital, and the Universities of São Paulo, Rio de Janeiro, and Rio Grande do Sul, as well as the Pontifical University of Rio Grande do Sul (PUCRS). Under the sponsorship of the Albert Einstein Hospital, Sirio-Libanês Hospital, CNPq, CAPES, FAPESP, FAPERJ, and the Department of Science and Technology of the Ministry of Health (DECIT), over 150 attendees, mostly principal investigators, i.e., leaders of research groups, discussed several aspects of cancer research, either in high-quality seminars and oral presentations or during poster sessions, where 77 posters were presented. This was a small, though highly focused, group.

As the Brazilian groups interacted and became up-todate with what was going on in each institution, foreign guests introduced some cutting-edge research to the forum.

However, this meeting had as one of its main goals the identification of the major challenges in implementing competitive cancer research in Brazil, as well as in successfully translating it to the clinical setting. If successful, translational research can effectively shorten the time it takes for patients to benefit from the most recent alternative treatments developed for their tumors. One session of the meeting was designed especially to focus on this issue. It featured the following guest speakers: Dirceu Barbano, President of the National Health Surveillance Agency (which is the Brazilian equivalent of the U.S. Food and Drug Administration); Antonio Campos de Carvalho, the head of DECIT; Jorge Venancio, coordinator of the National Ethics Committee (CONEP); and Dr. Carlos Gil, as a representative of the National Network for Development and Innovation in Anti-Cancer Drugs (REDEFAC).

The difficult process of importing reagents and drugs is one of the major problems faced by the scientific community in Brazil. It is almost impossible to be competitive whilst having to wait months for the release of imported goods and being required to pay three times the international market price when obtaining products through local representatives, in part because of the taxation system. Even if Brazilians were technically able to reproduce the cuttingedge treatments being developed abroad, such treatments would already be obsolete by the time the necessary reagents were obtained. This discussion, and any debate on the possible approaches to solve such problems, was stopped short due to the absence of representatives from the authorities that regulate this process, who were invited to the meeting.

Ideally, an alternative to the import of reagents would be to produce our own. This would not only make our science competitive but would also implement important changes in our economy - and that is where budgetary issues forestall our work. Antonio Campos de Carvalho, head of DECIT, spoke on this question (via the Internet because of a scheduling conflict). DECIT is a department within the Ministry of Health that coordinates basic, preclinical, and clinical research. The Ministry of Health has increased its investment in clinical research, including

Correspondence: A.P. Lepique: <alepique@icb.usp.br>.

Received July 11, 2014. Accepted October 24, 2014. First published online January 13, 2015. 
cancer research. Currently, there is a major deficit in the balance of trade in the technology and medicine sectors. In 2013 , Brazil had a $\mathrm{R} \$ 9.6$ billion deficit in this area. The MCT (Ministry of Science and Technology) aims to reduce this deficit with investments in this field. However, funding is still limited, amounting to $\mathrm{R} \$ 121$ million (approximately 60 million US dollars) in 2013. In cancer research in particular, funding reached $\mathrm{R} \$ 44$ million (about 20 million US dollars) for 190 projects, none of which has resulted in health innovation strategies. According to Campos de Carvalho, total funding would need to be 10 times higher than the actual amount for it to start making a difference to the status of cancer research in Brazil. Although we should appreciate that Brazil has a free and universal health system that, appropriately, receives the largest share of the total investment in healthcare, the United States invests eight billion US dollars and Europe invests three billion US dollars per year in oncology research. The average cost for each new drug development is 100 million US dollars over 10 years, and for kits and reagents for disease diagnosis it is approximately 50 million US dollars over 5 years. This is another area where there is an identified demand in Brazil, including for the development of diagnostic kits that could be used in the field, given that there are still several geographical regions that are difficult to access. Moreover, we do not yet have an implemented national program for the identification of molecular markers to predict responses to new-generation drugs. Such a program is very important so as to restrict the use of such drugs to those patients who will most likely benefit from treatment, thereby saving resources and lowering the overall cost of patient treatment.

A new oncology program was also recently launched called PRONON, the National Program for Supporting Oncology. This program brings together social institutions, non-profit organizations, and tax-saving programs and should reach $\mathrm{R} \$ 670$ million in 2014 . The program includes human resources training, research, and patient treatment. A potential problem with this program is that it may simply cause funding to switch from one type of research to another, for instance from basic to clinical research, thus missing out the intermediate sector - the technological research that leads to the creation and development of new therapies and diagnostics from work carried out in Brazil.

Clinical and biomedical cancer research had been increasing in Brazil, peaking in 2011, when 91 clinical studies were conducted, 83 of which were financed by industry. Our publications in this area ranked 15th in the world.

Because cancer impacts on population mortality and disability, it is also economically relevant, on account of the creation of jobs for highly trained personnel, the provision of patient access to new anti-cancer drugs, and research and development. It is worth pointing out that clinical research increases the expertise of medical and scientific groups, promotes associations between public and private organizations, and offers patients access to state-of-the-art therapies for free.

Carlos Gil addressed one of the positive initiatives of recent years, the development of a network for anti-cancer drugs in Brazil, REDEFAC (National Network for Development and Innovation in Anti-Cancer Drugs). REDEFAC is a nationwide program that supports innovation in oncology drug development. The network is coordinated by the Brazilian Ministry of Health, which has strategically designated INCA's clinical research team to spearhead and execute the program along with nonclinical researchers in Brazil. The idea is to boost translational research on experimental products that will be licensed to biotech investors or pharmaceutical companies that are committed to maintaining their developmental framework. These homedeveloped technologies will then have a foreseeable progression to the market, with the guaranteed option of counting on INCA's world-class clinical research facilities for human trials. To achieve its ambitious goal of entering competitively into the oncology research market, REDEFAC has teamed up with important partners in Brazil, such as the Brazilian Development Bank, the Agency for Funding Innovation, the Oswaldo Cruz Foundation, the National Laboratory of Biosciences, and INCA. Involvement with the private sector is considered strategic, and REDEFAC's first milestone is to provide investors and pharmaceutical companies with a pipeline of developing oncology products for their choice of investment; hence, partnerships with the private sector are highly important in the early stages of development, and our team is constantly available to discuss any possible venture. In line with REDEFAC, the Ministry of Health implemented in 2014 the National Network of Clinical Research in Cancer to balance preclinical and clinical demands within the country.

Last, but not least, Brazilian researchers in basic and clinical areas have to deal with one more major hurdle: the approval of clinical trials by the National Ethics Committee CONEP. Carlos Barrios, from PUCRS, pointed out that, while the average time for protocol approval in other countries is 3 to 8 months, in Brazil it is 14 months. In the United States, where society has realized what a major part of the economy this industry can be, the time to start a clinical trial can be as short as 3 months. This means that Brazil is missing out on industry-financed projects, because when we finally get CONEP's approval, other countries have actually finished recruiting patients for the trial, or have even finished the trial itself. Dr. Gustavo Steffanoff represented CONEP in the meeting and pointed out that there was a $100 \%$ increase in the number of projects approved by CONEP from 2012 to 2013. There are initiatives to increase CONEP's efficiency, including the hiring of more staff to review and allocate new projects, as well as remodeling of the offices. However, there is still a backlog of new protocols accumulating in CONEP, taking too long to obtain a response, negative or positive. Today, there are 683 institutional Ethics Committees registered at 
CONEP. According to the data in CONEP's Plataforma Brazil (an online platform comprising all data regarding clinical trials in the country), there were 185,000 projects registered in 2013 in 90,000 institutions (total numbers, not specifically related to cancer research). Still, this is a small number considering the size of the Brazilian population and the scale of cancer incidence.

Such is the status of our field in Brazil today. We can follow and marvel at the advances that our colleagues make in other countries, but we are stuck. We cannot be competitive or develop national solutions without addressing these time-consuming regulatory and bureaucratic issues. We need the government to follow through and increase research funding, at the same time facilitating the regulatory processes and maintaining high ethical standards at all times. Brazilian scientists understand that cancer research is much bigger than academia. It constitutes a major economic stimulus that generates jobs, wealth, and technological independence, because in essence it is innovative. Perhaps what all these scattered skills need are leadership and organizational guidance. Federal administration is the most obvious choice for the top job; however, without sizeable budgets committed long-term and strict timeframes for government authorities to mitigate conflicts of interest at the public-private interface and to clear away excessive regulations/bureaucracy, we risk getting stuck or, even worse, moving in a reverse direction towards stagnation.

\section{Acknowledgments}

We would like to thank Dr. Martin Bonamino, from Instituto Nacional do Câncer in Rio de Janeiro, RJ, and Prof. Deilson Elgui de Oliveira, from Universidade Estadual Paulista, Botucatu, SP, for their valuable contributions in revising this article. 